\title{
NOUVELLE
}

\section{Stimulation cérébrale profonde du striatum ventral dans le traitement du trouble obsessionnel-compulsif avec dépression majeure}

Bruno Aouizerate, Corinne Martin-Guehl, Emmanuel Cuny, Dominique Guehl, Hélène Amieva, Abdelhamid Benazzouz, Colette Fabrigoule, Michele Allard, Alain Rougier, Pierre Burbaud, Jean Tignol, Bernard Bioulac

$>$ Le trouble obsessionnel-compulsif (TOC) est une affection psychiatrique relativement fréquente avec une prévalence «vie entière » de l'ordre de $2 \%$ à $3 \%$ en population générale [1]. II est souvent associé à la dépression majeure et représente un des troubles anxieux les plus invalidants [2] de par l'intensité des symptômes qui le caractérise et la gêne fonctionnelle qu'il entraîne. Heureusement, la recherche et notamment l'enrichissement permanent de nos connaissances sur les relations structurefonction, ainsi que l'apport récent de la neuro-imagerie, permettent actuellement de mieux appréhender la physiopathologie de ce trouble et de proposer une hypothèse essentiellement fondée sur l'approche anatomo-fonctionnelle.

\section{Aspects physiopathologiques :} approche anatomo-fonctionnelle

\section{Bases phénoménologiques}

Les obsessions sont définies par l'irruption intrusive et incessante dans la pensée d'une idée, d'une impulsion ou d'une représentation. Les compulsions sont des comportements répétitifs qui traduisent, en général, la lutte contre les obsessions, visant à réduire la charge anxieuse résultant de leur émergence. Ces définitions conduisent à prendre en compte, au cœur de la symptomatologie obsessionnelle-compulsive, cette impression fondamentale pour l'individu que «quelque chose ne va pas » (something is wrong [3]). En d'autres termes, les obsessions peuvent être conçues par le sujet comme la perception permanente d'être en faute et/ou en situation d'erreur. Les compulsions apparaissent comme des réponses comportementales destinées à soulager la tension engendrée par l'irruption des pensées obsédantes. Les conduites compulsives ont ainsi pour but de mettre fin aux signaux de faute et/ou d'erreur que le sujet perçoit. Il est alors amené à reproduire «en boucle» ces comportements sur la base d'un état émotionnel et motivationnel interne orienté vers l'obtention d'un soulagement durable, assimilable à une forme de récompense. Ces aspects phénoménologiques suggèrent donc l'altération d'un certain nombre de fonctions dans le TOC, qu'il s'agisse de la détection des erreurs, des processus émotionnels, motivationnels et de récompense $[4,5]$.

\section{Neurophysiologie expérimentale}

Cette approche phénoménologique du TOC suggère le rôle potentiel des circuits cortico-striato-pallido-thalamo-corticaux dans la physiopathologie de cette affection. Parmi ceux-ci, les circuits trouvant leur origine au niveau du cortex orbitofrontal (COF) et du cortex cingulaire antérieur (CCA) ont été proposés comme étroitement impliqués dans la production des symptômes obsessionnels-compulsifs à la lumière des données de la neurophysiologie expérimentale centrées sur l'analyse des relations structure-fonction.

Le COF semble participer à plusieurs fonctions: (1) il joue un rôle essentiel dans la saisie des informations émanant de l'environnement en leur conférant un sens, une signification sur un plan émotionnel et motivationnel tenant compte des expériences antérieures du sujet, et sous-tendant la prise de décision [4-6] ; (2) il intervient également dans des aspects plus cognitifs comme la détection des erreurs [3] ou encore la sélection, le jugement et la comparaison des stimulus environnementaux [7].

Le CCA est impliqué aux côtés du COF, dans divers processus, tant cognitifs (attention, mémoire de travail, prédiction de l'erreur, détection des erreurs associée à une activation neuronale chez le primate lors de paradigmes expérimentaux le plaçant potentiellement en situation d'erreur, gestion des situations de conflits, sélection de réponses et anticipation), qu'émotionnels et motivationnels $[4,5,8]$.

Le striatum dorsal se voit investi, sur la base d'observations cliniques, d'un rôle majeur dans l'initiation et la programmation de profils comportementaux routiniers et/ou d'habitude $[9,10]$. Néanmoins, dans sa région ventrale, le 
striatum intervient davantage, par ses connexions anatomiques privilégiées avec les COF et CCA, dans les réponses orientées vers l'obtention d'une récompense, notamment lors de la préparation, l'initiation et l'exécution du comportement [6]. Il exerce également, en lien avec les afférences dopaminergiques méso-limbiques [11], une fonction centrale dans l'évaluation des conséquences possibles de l'action ou «critique », permettant à l'«acteur» d'ajuster son comportement de façon à répondre au but ou objectif fixé si l'on fait référence au modèle « acteurcritique » de Sutton et Barto [12].

\section{Neuro-imagerie fonctionnelle}

Les données de la neuro-imagerie n'ont fait que conforter davantage l'hypothèse d'un rôle prédominant des circuits orbitofrontal et cingulaire antérieur dans l'émergence des symptômes obsessionnels-compulsifs. Les recherches dans le domaine fonctionnel ont permis de montrer que plusieurs régions cérébrales présentaient une activité anormalement élevée chez les patients atteints de TOC, qu'il s'agisse de la région orbitale du cortex préfrontal, du CCA, du striatum (noyau caudé), ou du thalamus $[4,5$, 13]. Cette hyperactivité est observée à la fois dans des conditions de base, ou lors de situations expérimentales consistant à provoquer les manifestations obsessionnelles-compulsives $[4,5,9,13]$. Une activité fonctionnelle plus importante au sein du CCA a été également rapportée chez les patients avec TOC lors de tâches comportementales les amenant à réaliser des erreurs possibles [14, 15].

L'ensemble des arguments expérimentaux précités suggère donc le rôle déterminant d'un dysfonctionnnement des circuits cortico-striato-pallido-thalamo-corticaux prenant naissance tant au niveau du COF que du CCA dans la pathogénie du TOC.

\section{Aspects thérapeutiques : place} de la stimulation cérébrale profonde L'efficacité des traitements antidépresseurs et des thérapies cognitivo-com- portementales est aujourd'hui clairement établie dans la prise en charge du TOC. Néanmoins, ces stratégies s'avèrent peu ou pas efficaces dans $25 \%$ à $30 \%$ des cas [16]. Diverses approches neurochirurgicales ont alors été utilisées, dont la réalisation de lésions bilatérales de la capsule antérieure, zone de passage des fibres reliant le thalamus aux aires corticales limbiques représentées par les COF et CCA [16]. Ces techniques neurochirurgicales se sont révélées efficaces

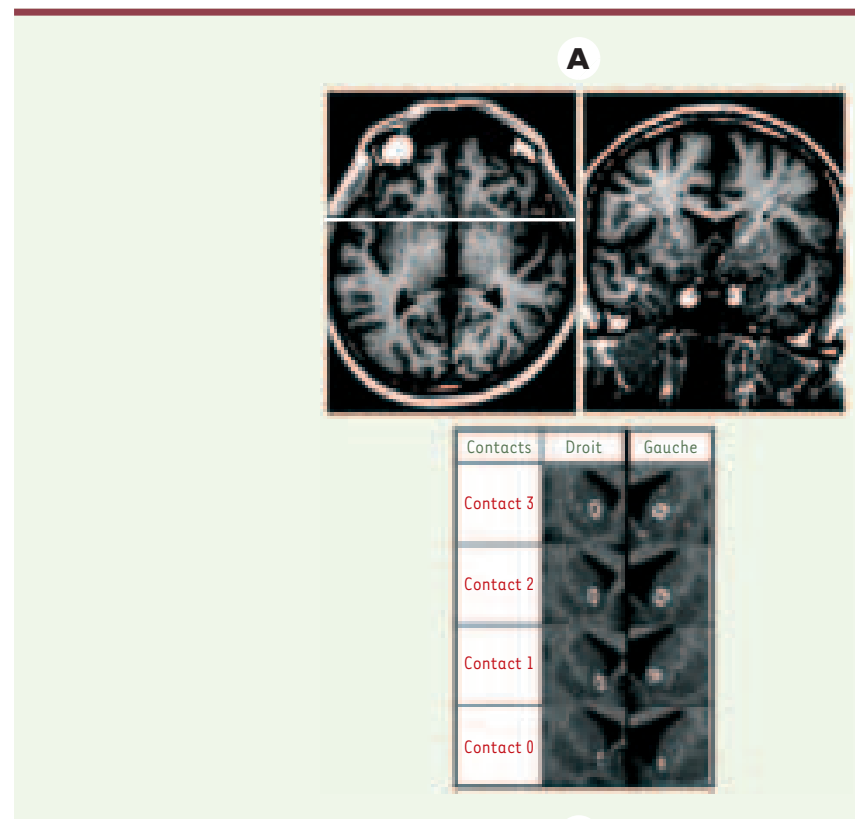

B
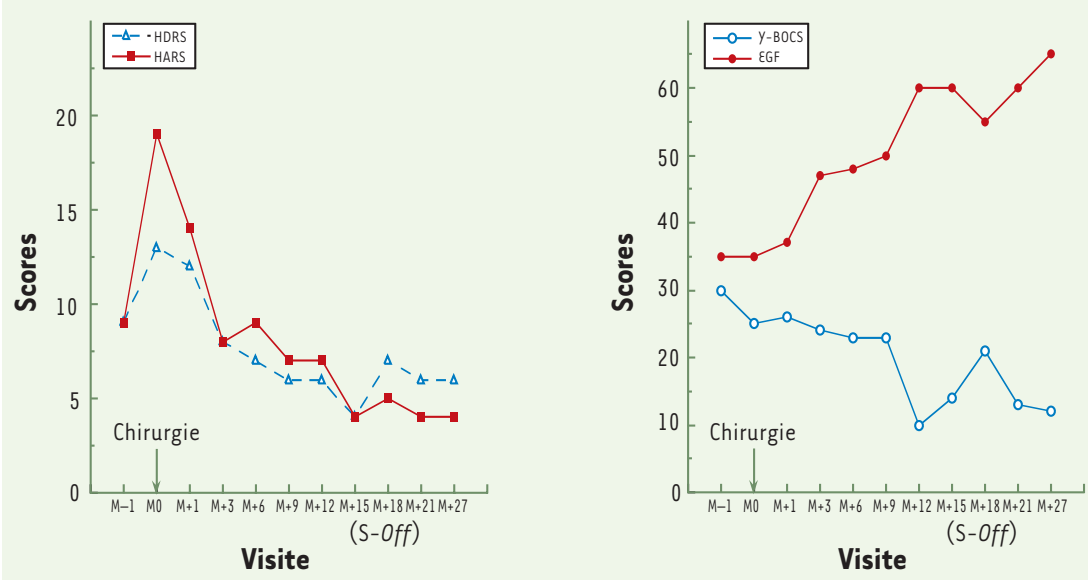

Figure 1. A. Coupe axiale (en haut et à gauche) et coupe coronale (en haut et à droite) montrant l'implantation bilatérale des électrodes dans le striatum ventral. Coupes coronales (en bas) montrant la localisation des quatre plots de chacune des électrodes implantées au sein du striatum ventral ( $C$ : noyau caudé ; $P$ : putamen; acc : noyau accumbens ; $A C$ : capsule antérieure). B. Illustration des effets de la stimulation cérébrale profonde sur les symptômes dépressifs (HDRS) et anxieux (HARS) (à gauche), sur les symptômes obsessionnels-compulsifs (Y-BOCS) et le fonctionnement global ( $\varepsilon G F)$ (à droite) ( $M-1$ : sous traitement pharmacologique ; $M 0$ : après arrêt du traitement pharmacologique ; $M+1, M+6, M+9, M+12, M+15, M+18, M+21, M+27$ : après 1 , $3,6,9,15,18,21$ et 27 mois de stimulation cérébrale profonde ; $S$-off : stimulateur non fonctionnel). 
dans $60 \%$ à $70 \%$ des cas, mais elles sont aujourd'hui bien moins pratiquées car les lésions causées sont irréversibles et peuvent entraîner des complications d'ordre cognitif et émotionnel (euphorie, agitation psychomotrice, émoussement affectif, aspontanéité motrice...).

La stimulation cérébrale profonde (SCP) de la capsule antérieure a été testée pour remplacer cette chirurgie lésionnelle. Cette technique, qui consiste en l'implantation d'électrodes au niveau de la région cérébrale d'intérêt, a été efficace chez trois des quatre patients opérés présentant un TOC [17]. Néanmoins, les paramètres de stimulation utilisés dans cette étude nous ont amené à considérer les régions bordant la capsule antérieure comme cible de choix pour la SCP. Parmi ces régions d'intérêt, la partie ventro-médiane du striatum (incluant la tête du noyau caudé et le noyau accumbens) (Figure $1 A$ ) nous a semblé particulièrement intéressante au vu des connaissances que nous avons également de la physiopathologie du TOC abordée précédemment $[4,5,18,19]$.

Nous avons pu mettre en évidence chez un patient souffrant d'un TOC sévère et chronique, une réduction importante de la sévérité des symptômes dépressifs et anxieux dans les trois premiers mois de $S C P$, avec l'obtention d'une rémission après six mois. Les effets favorables sur le TOC se sont révélés plus tardifs avec une amélioration franche des symptômes et une rémission observées dès le $12^{\mathrm{e}}$ mois de traitement, perdurant lors de l'évaluation psychométrique pratiquée trois mois plus tard [20]. Vers le $17^{\mathrm{e}}$ mois de suivi post-opératoire, une recrudescence de la symptomatologie obsessionnellecompulsive est alléguée par le patient, ce que vient confirmer l'évaluation psychométrique réalisée dans les suites qui, bien que ne montrant pas de réelle aggravation de la sévérité des symptômes dépressifs et anxieux, révéla des scores de sévérité des symptômes obsessionnels-compulsifs revenus quasiment aux niveaux initiaux. La vérification du fonctionnement du stimulateur a permis de mettre en évidence une panne de ce dernier. Une fois changé, un retour de l'amélioration à son niveau optimal a été observé dans les 3 mois suivants, ainsi qu'au $27^{\mathrm{e}}$ mois de suivi [21] (Figure 1B). Fait important, aucune altération des tests neuropsychologiques ni aucun effet secondaire clinique n'ont été retrouvés [20, 21].

Ces résultats sont d'une grande importance dans la mesure où ils permettent de conforter le rôle des boucles fronto-sous-corticales mettant en jeu le striatum ventral dans la production des manifestations obsessionnelles-compulsives et dépressives. Même si ces résultats doivent être confirmés chez un nombre plus important de patients, ils apparaissent prometteurs car cette région sous-corticale pourrait représenter une cible de choix pour traiter par SCP des formes de TOC et dépression majeure rebelles aux démarches thérapeutiques usuelles. $\diamond$

Deep brain stimulation of the ventral

striatum in the treatment of

obsessive-compulsive disorder

and major depression

\section{RÉFÉRENCES}

1. Antony MM, Downie F, Swinson RP. Diagnostic issues and epidemiology in obsessive-compulsive disorder. In : Swinson RP, Antony MM, Rachman S, Richter MA, eds. Obsessive-compulsive disorder. Theory, research, and treatment. New York : The Guilford Press, 1998 : 3-32.

2. Koran LM, Thienemann ML, Davenport R. Quality of life for patients with obsessive-compulsive disorder. Am J Psychiatry 1996 ; 153 : 783-8.

3. Schwartz JM. A role of volition and attention in the generation of new brain circuitry. Toward a neurobiology of mental force. J Consciousness Studies $1999 ; 6: 115-42$
4. Aouizerate B, Guehl D, Cuny $\varepsilon$, et al. Pathophysiology of obsessive-compulsive disorder: a necessary link between phenomenology, neuropsychology, imagery and physiology. Prog Neurobiol 2004 ; 72 : 195-221.

5. Aouizerate B, Guehl D, Cuny $\varepsilon$, et al. Updated overview of the putative role of serotoninergic system in obsessive-compulsive disorder. Neuropsychiatr Dis Treat $2005 ; 1: 231-43$.

6. Tremblay L, Schultz W. Relative reward preference in primate orbitofrontal cortex. Nature $1999 ; 398$ : 704-8.

7. Ramnani N, Owen AM. Anterior prefrontal cortex: insights into function from anatomy and neuroimaging. Nat Rev Neurosci 2004 ; 5 : 184-94.

8. Brown JW, Braver TS. Learned predictions of error likelihood in the anterior cingulate cortex. Science 2005 ; 307 : 1118-21.

9. Baxter LR. Functional imaging of brain systems mediating obsessive-compulsive disorder: clinical studies. In: Charney DS, Nestler $\varepsilon$ J, Bunney BS, eds. Neurobiology of mental illness. New York : Oxford University Press, 1999 : 534-47.

10. Jog MS, Kubota Y, Connolly Cl, et al. Building neural representations of habits. Science $1999 ; 286$ : 1745-9.

11. O'Doherty J, Dayan P, Schultz J, et al. Dissociable roles of ventral and dorsal striatum in instrumental conditioning. Science 2004 ; 304 : 452-4.

12. Sutton RS, Barto AG. Reinforcement learning: an introduction. Cambridge, Massachusetts : MIT Press, 1998.

13. Saxena S, Brody AL, Schwartz JM, Baxter LR. Neuroimaging and frontal-subcortical circuitry in obsessive-compulsive disorder. BrJ Psychiatry 1998; 35 (suppl) : 26-37.

14. Fitzgerald KD, Welsh RC, Gehring WJ, et al. Error-related hyperactivity of the anterior cingulate cortex in obsessive-compulsive disorder. Biol Psychiatry 2005; $57: 287-94$.

15. Maltby N, Tolin DF, Worhunsky P, et al. Dysfunctional action monitoring hyperactivates frontal-striatal circuits in obsessive-compulsive disorder: an eventrelated fMRI study. Neuroimage 2005 ; 24 : 495-503.

16. Jenike MA. Neurosurgical treatment of obsessivecompulsive disorder. BrJ Psychiatry 1998; 35 (suppl) : $79-90$.

17. Nuttin B, Cosyns P, Demeulemeester $H$, et al. Electrical stimulation in anterior limbs of internal capsules in patients with obsessive-compulsive disorder. Lancet $1999 ; 354: 1526$.

18. Laplane D. Obsessive-compulsive disorders caused by basal ganglia diseases. Rev Neurol (Paris) 1994 ; 150 : 594-8.

19. Laplane D, Levasseur M, Pillon B, et al. Obsessivecompulsive and other behavioural changes with bilateral basal ganglia lesions. A neuropsychological, magnetic resonance imaging and positron tomography study. Brain 1989; 112: 699-725.

20. Aouizerate B, Cuny $\varepsilon$, Martin-Guehl C, et al. Deep brain stimulation of the ventral caudate nucleus is effective in obsessive-compulsive disorder and major depression. Case report. J Neurosurg 2004 ; 101 : 482-6.

21. Aouizerate B, Martin-Guehl C, Cuny $\varepsilon$, et al. Deep brain stimulation for obsessive-compulsive disorder and major depression. Am J Psychiatry 2005 (sous presse). 